\title{
Genetic Divergence for Important Economic and Quality Traits in Improved Lines of Rice (Oryza sativa $\mathrm{L}$.)
}

\author{
Aditya Bora, Dhanraj Meena*, Mukesh Kumar Karnwal and Himanshu Chaudhary
}

Department of Genetics and Plant Breeding, College of Agriculture G.B. Pant University of Agriculture and Technology, Pantnagar-263145(U.S.Nagar, Uttarakhand), India

*Corresponding author

\section{A B S T R A C T}

\begin{tabular}{l} 
Key w or d s \\
$\begin{array}{l}\text { Rice (Oryza sativa } \\
\text { L.), Mahalanobis D } \\
\text { analysis, Genetic } \\
\text { divergence and } \\
\text { Cluster }\end{array}$ \\
\hline Article Info \\
$\begin{array}{l}\text { Accepted: } \\
\text { 24 August } 2020 \\
\text { Available Online: } \\
\text { 10 September } 2020\end{array}$ \\
\hline
\end{tabular}

\section{Introduction}

Rice is the seed of the grass species Oryza sativa (Asian rice) or Oryza glaberrima (African rice). As a cereal grain, it is the most widely consumed staple food for a large part of the world's human population, especially in Asia. It is the agricultural commodity with the third-highest worldwide production (rice, 741.5 million tons in 2014), after sugarcane (1.9 billion tons) and maize (1.0 billion tons).Major rice producing countries India, China, Indonesia, Thailand and Bangladesh make up a little over two-thirds of total area under rice. The World Rice Production in 2017/18 will be 481.04 million metric tons, which is 2.06 million tons less than the last year production i.e. 483.1 million tons (USDA). In India, estimated rice production in $2016 / 17$ is 105 million tons from 44 million hectares as compared to 103.5 million tons harvested from 43.46 million hectares in 2015-16. India is $2^{\text {nd }}$ largest rice producing country in the world after China (145MT). Rice cultivation is well-suited to countries and regions with low labor costs and high 
rainfall, as it is labor-intensive to cultivate and requires ample water. However, rice can be grown practically anywhere, even on a steep hill or mountain area with the use of water-controlling terrace systems. The varieties of rice are typically classified as long, medium, and short-grained. The grains of long-grain rice (high in amylose) tend to remain intact after cooking, medium-grain rice (high in amylopectin) becomes stickier and short grain rice contents low amylose.

Genetic diversity and the diverse gene pools are the basis of plant breeding. In addition to new gene pool providing building blocks for further improvement, genetic diversity is essential if higher level of productivity is to be achieved and sustained during the process of varietal development. The more diverse the parents, within overall limits of fitness, greater are the chances of obtaining higher amount of heterotic expression in $\mathrm{F}_{1} \mathrm{~s}$ and broad spectrum of variability in segregating generations(Singh and Bains 1968).Thus genetic divergence among parents is of paramount importance in selecting parental genotype for crossing programmes. The use of Mahalanobis $\mathrm{D}^{2}$ statistic for estimating genetic divergence has been emphasized by Shukla et al., (2006), Sarawgi and Binse, 2007 and (Ramya and Senthilkumar, 2008). Hence the present investigation was carried out to assess the nature and magnitude of genetic diversity among the genotypes for further utilization in breeding programmes.

\section{Materials and Methods}

The experimental material for the present study comprised of 28 genotypes of rice laid in randomized complete block design (RCBD) with 3 replications at the Norman E. Borlaug Crop Research Centre, GovindBallabh Pant University of Agriculture and Technology, Pantnagar, Uttarakhand, during two seasons kharif 2012 and kharif
2013. Observations were recorded on randomly selected plants per replication for days to $50 \%$ flowering, days to maturity, plant height $(\mathrm{cm})$, panicle length $(\mathrm{cm})$, number of panicle per plant, number of tillers, number of grains per panicle, flag leaf length $(\mathrm{cm})$, flag leaf breadth $(\mathrm{cm})$, flag leaf area (sq. $\mathrm{cm})$, 1000 grain weight $(\mathrm{g})$, grain yield per plant $(\mathrm{g})$, dry weight of plant $(\mathrm{g})$, harvest index. (\%), decorticated grain length (mm), decorticated grain breadth $(\mathrm{mm})$, decorticated $\mathrm{L} / \mathrm{B}$ ratio, gelatinization temperature through alkali spreading value and Endosperm: amylosecontent. The data was subjected to Mahalanobis $\mathrm{D}^{2}$ statistics to measure the genetic divergence as suggested by Rao (1952).

\section{Results and Discussion}

By using Tocher's methods the twenty eight genotypes were grouped into six clusters in both the seasons (kharif 2012 and 2013) and over the seasons. Cluster I during 2013 and in pooled estimates was the largest cluster having 12 and 9 genotypes; whereas cluster III during 2012 (13 genotypes) was largest. Cluster III in 2013, Cluster V in both the seasons $(2012,2013)$ and over the seasons were monogenotypic cluster. Cluster IV in both the seasons and cluster V in 2013 had five genotypes each. Cluster II in 2012 and cluster $\mathrm{V}$ in over the seasons had 6 genotypes each. Cluster II, III, and IV in pooled estimates had 4 genotypes each. Cluster I in 2012 had two genotypes and cluster II in 2013 had 3 genotypes (Table 1). In 2012 the maximum inter-cluster $\mathrm{D}^{2}$ values were obtained between clusters I and IV (74.537), followed by cluster I and VI (74.289). During 2013, cluster II and III (79.081) followed by cluster III and V (76.819); whereas in pooled estimates cluster IV and V (50.044) followed by cluster I and V (46.633) had maximum inter-cluster $\mathrm{D}^{2}$ values. Parental lines selected from these three clusters may be used in a 
hybridization programme, since hybridization between divergent parents is likely to produce wide variability and transgressive segregations with high heterotic effects. Such recommendations were also made by Murty and Arunachalam (1966), Rao and Gomanthinayagam (1997) Mishra et al., (2003), Chaturvedi and Maurya (2005) and Sandhya Kishore et al., (2007).

Table. 1 Cluster Pattern of 28 Genotypes of Rice (On The Basis Of $D^{2}$ Values)

\begin{tabular}{|c|c|c|c|}
\hline $\begin{array}{l}\text { Cluster } \\
\text { number }\end{array}$ & Year & Genotypes included & $\begin{array}{l}\text { Number of } \\
\text { genotypes }\end{array}$ \\
\hline \multirow[t]{3}{*}{ I } & 2012 & C 1446-5-18-17-1-MLD 2, CR-2995-1-2-3-1-1 & 2 \\
\hline & 2013 & $\begin{array}{l}\text { C 1446-5-18-17-1-MLD 2, NLR- 40024, JGL-17183, } \\
\text { UPRI-2009-9, NP-6226, R-1570-2644-2-1547, CR- } \\
\text { 2995-1-2-3-1-1, PR-113, CR-77-3-1-1, NP-218, } \\
\text { KAGR-559-1, Pant Dhan-12 }\end{array}$ & 12 \\
\hline & Pooled & $\begin{array}{l}\text { C 1446-5-18-17-1-MLD 2, JGL-17183, WGL- 365, } \\
\text { UPR-3411-1-1-1, UPR-3305-6-1-2, CR-77-3-1-1, NP- } \\
\text { 107-5, GontraBidhan -3, UPR-3027-10-1-1 }\end{array}$ & 9 \\
\hline \multirow[t]{3}{*}{ II } & 2012 & $\begin{array}{l}\text { NLR- 40024, UPRI- 3425-14-3-1, UPR-3411-1-1-1, } \\
\text { Pant Dhan-12, UPR-3027-10-1-1, Pant Dhan-4 }\end{array}$ & 6 \\
\hline & 2013 & OR 2324-25-1, UPRI- 3425-14-3-1, UPR-3305-6-1-2 & 3 \\
\hline & Pooled & $\begin{array}{l}\text { NLR- 40024, UPRI-2009-9, UPR-2805-14-1-2, NP- } \\
6226\end{array}$ & 4 \\
\hline \multirow[t]{3}{*}{ III } & 2012 & $\begin{array}{l}\text { JGL-17183, OR 2324-25-1, UPR-3443-7-2-1, WGL- } \\
\text { 365, PAU-3832-79-4-3-1, UPR-3305-6-1-2, R 1576- } \\
\text { 1700-1-560-1, CR-77-3-1-1, KJT-1-11-15-23-26-22, } \\
\text { NP-218, KAGR-559-1, NP-107-5, GontraBidhan -3 }\end{array}$ & 13 \\
\hline & 2013 & UPR-2805-14-1-2 & 1 \\
\hline & Pooled & $\begin{array}{l}\text { OR 2324-25-1, R-1570-2644-2-1547, Pant Dhan-12, } \\
\text { Pant Dhan-18 }\end{array}$ & 4 \\
\hline \multirow[t]{3}{*}{ IV } & 2012 & $\begin{array}{l}\text { UPRI-2009-9, NP-6226, JGL- 17196, R-1570-2644- } \\
\text { 2-1547, PR-113 }\end{array}$ & 5 \\
\hline & 2013 & $\begin{array}{l}\text { UPR-3443-7-2-1, WGL- 365, JGL- 17196, PAU- } \\
\text { 3832-79-4-3-1, UPR-3027-10-1-1, Pant Dhan-18 }\end{array}$ & 5 \\
\hline & Pooled & $\begin{array}{l}\text { UPRI- 3425-14-3-1，UPR-3443-7-2-1，NP-218, } \\
\text { KAGR-559-1 }\end{array}$ & 4 \\
\hline \multirow[t]{3}{*}{$\mathbf{V}$} & 2012 & UPR-2805-14-1-2 & 1 \\
\hline & 2013 & $\begin{array}{l}\text { UPR-3411-1-1-1, R 1576-1700-1-560-1, KJT-1-11- } \\
\text { 15-23-26-22, NP-107-5, GontraBidhan -3 }\end{array}$ & 5 \\
\hline & Pooled & $\begin{array}{l}\text { JGL- 17196, PAU-3832-79-4-3-1, R 1576-1700-1-560- } \\
\text { 1, CR-2995-1-2-3-1-1, PR-113, KJT-1-11-15-23-26-22 }\end{array}$ & 6 \\
\hline \multirow[t]{3}{*}{ VI } & 2012 & Pant Dhan-18 & 1 \\
\hline & 2013 & Pant Dhan-4 & 1 \\
\hline & Pooled & Pant Dhan-4 & 1 \\
\hline
\end{tabular}


Table.2 Averages Inter and Intra-Cluster (Diagonal) $\mathrm{D}^{2}$ values in rice genotypes

\begin{tabular}{|c|c|c|c|c|c|c|c|}
\hline Cluster & Year & I & II & III & IV & V & VI \\
\hline \multirow[t]{3}{*}{ I } & 2012 & 10.692 & 64.250 & 57.761 & 74.537 & 26.868 & 80.288 \\
\hline & 2013 & 45.769 & 42.463 & 43.882 & 14.937 & 39.024 & 18.733 \\
\hline & pooled & 28.702 & 21.231 & 26.357 & 7.984 & 46.633 & 29.671 \\
\hline \multirow[t]{3}{*}{ II } & 2012 & & 32.440 & 11.411 & 15.297 & 58.117 & 19.583 \\
\hline & 2013 & & 17.549 & 79.081 & 28.896 & 11.391 & 35.120 \\
\hline & pooled & & 50.256 & 13.348 & 23.597 & 29.259 & 20.415 \\
\hline \multirow[t]{3}{*}{ III } & 2012 & & & 39.238 & 17.909 & 52.859 & 26.082 \\
\hline & 2013 & & & 0 & 54.148 & 76.819 & 57.983 \\
\hline & pooled & & & 36.562 & 28.595 & 26.015 & 8.445 \\
\hline \multirow[t]{3}{*}{ IV } & 2012 & & & & 28.069 & 66.439 & 19.695 \\
\hline & 2013 & & & & 40.112 & 25.182 & 14.182 \\
\hline & pooled & & & & 35.995 & 50.044 & 32.087 \\
\hline \multirow[t]{3}{*}{$\mathbf{V}$} & 2012 & & & & & 0 & 74.289 \\
\hline & 2013 & & & & & 25.669 & 31.552 \\
\hline & pooled & & & & & 32.178 & 28.405 \\
\hline \multirow[t]{3}{*}{ VI } & 2012 & & & & & & 0 \\
\hline & 2013 & & & & & & 0 \\
\hline & pooled & & & & & & 0 \\
\hline
\end{tabular}


Table.3 Cluster mean values for different characters in rice

\begin{tabular}{|c|c|c|c|c|c|c|c|c|c|c|c|c|c|c|c|c|c|c|c|c|}
\hline Clusters & Season & $\begin{array}{c}\text { Days To } \\
\mathbf{5 0} \% \\
\text { Flowering }\end{array}$ & $\begin{array}{l}\text { Days To } \\
\text { Maturity }\end{array}$ & $\begin{array}{l}\text { Plant } \\
\text { Height } \\
\text { (cm) }\end{array}$ & $\begin{array}{c}\text { Panicle } \\
\text { Length } \\
(\mathbf{c m})\end{array}$ & $\begin{array}{l}\text { No of } \\
\text { tillers }\end{array}$ & \begin{tabular}{|c|} 
No of \\
productiy \\
e tillers
\end{tabular} & $\begin{array}{l}\text { No of } \\
\text { Grain/ } \\
\text { Panicle }\end{array}$ & $\begin{array}{c}\text { Leaf } \\
\text { Length } \\
(\mathrm{cm})\end{array}$ & $\begin{array}{c}\text { Leaf } \\
\text { Breadth } \\
(\mathrm{cm})\end{array}$ & $\begin{array}{c}\text { Flag Leal } \\
\text { Area } \\
\text { (sq.m) }\end{array}$ & $\begin{array}{c}\mathbf{1 0 0 0} \\
\text { Grain } \\
\text { Weight } \\
\text { (g) }\end{array}$ & $\begin{array}{c}\text { Grain } \\
\text { Yield/ } \\
\text { Plant } \\
\text { (g) }\end{array}$ & $\begin{array}{c}\text { Dry } \\
\text { Weight } \\
\text { (g) }\end{array}$ & $\begin{array}{c}\text { Harvest } \\
\text { Index } \\
(\%)\end{array}$ & $\begin{array}{c}\text { Grain } \\
\text { length } \\
(\mathbf{m m})\end{array}$ & $\begin{array}{c}\begin{array}{c}\text { Grain } \\
\text { breadth } \\
(\mathbf{m m})\end{array} \\
\text { (a) }\end{array}$ & L/b Ratio & $\begin{array}{c}\text { Alkali } \\
\text { Digestior }\end{array}$ & $\begin{array}{c}\text { Amylose } \\
\text { Content } \\
(\%)\end{array}$ \\
\hline \multirow[t]{3}{*}{ I } & 2012 & 97.670 & 127.670 & 94.300 & 25.630 & 8.870 & 9.000 & 258.630 & 33.730 & 1.600 & 40.650 & 19.870 & 11.930 & 50.730 & 23.530 & 6.000 & 1.800 & 3.340 & 3.760 & 20.250 \\
\hline & 2013 & 109.000 & 140.000 & 93.733 & 27.633 & 9.000 & 9.067 & 187.667 & 33.867 & 1.433 & 36.467 & 21.521 & 13.690 & 56.833 & 24.435 & 5.067 & 1.867 & 2.722 & 6.192 & 20.670 \\
\hline & pooled & 101.667 & 131.889 & 99.889 & 24.418 & 9.433 & 9.520 & 210.806 & 33.427 & 1.597 & 41.780 & 20.687 & 12.419 & 47.686 & 30.052 & 5.580 & 1.894 & & 5.322 & .546 \\
\hline \multirow[t]{3}{*}{ II } & 2012 & 101.000 & 131.000 & 101.340 & 24.560 & 8.930 & 9.000 & 194.700 & 34.200 & 1.670 & 10 & 20.280 & 12.960 & 50.670 & & 5.5 & 1.8 & & 5.640 & 100 \\
\hline & 2013 & 107.667 & 137.667 & 98.900 & 25.000 & 9.267 & 9.267 & 176.967 & 39.867 & 1.660 & 49.6 & 20.407 & 10.830 & 54.500 & & 5.6 & 1.9 & & 5.524 & .523 \\
\hline & pooled & 106.500 & 136.875 & 99.881 & 25.571 & 10.446 & 10.575 & 192.067 & 37.455 & 1.591 & 6.418 & 23.003 & 12.425 & 45.865 & & 5.6 & 1.9 & 956 & 5.046 & .321 \\
\hline \multirow[t]{3}{*}{ III } & 2012 & 110.000 & 141.000 & 95.930 & 26.810 & 9.330 & 9.400 & 195.330 & 35.6 & 1.610 & & & 13.190 & 57.8 & & 5.0 & & 600 & 130 & \\
\hline & 2013 & 98.333 & 129.333 & 104.433 & 25.133 & 10.467 & & 240.733 & 43.8 & & & & 12.690 & 41. & & 6.1 & & & 4.592 & .997 \\
\hline & pooled & 107.542 & 137.917 & 101.486 & 25.690 & 9.463 & & 187.025 & 32.775 & 1.549 & 37.551 & 24.299 & 14.091 & 51.419 & & 5.7 & 2.0 & 782 & 4.602 & 21.152 \\
\hline \multirow[t]{3}{*}{ IV } & 2012 & 111.330 & 141.3 & 102.340 & 25.690 & 8.5 & & 169.770 & 32.670 & & & & & 45.3 & & 5.2 & & & & \\
\hline & 2013 & 104.000 & 134.000 & 105.833 & 27.733 & 8.067 & 8.133 & 175.900 & 28.300 & 1.663 & & & & 50.833 & & 6.2 & 2.0 & 006 & 5.885 & 22.200 \\
\hline & pooled & 102.792 & 133.667 & 99.898 & 26.665 & 9.083 & 9.183 & 213.633 & 37.800 & 1.486 & 43.030 & 21.461 & 12.995 & 51.958 & 32.476 & 5.517 & 1.8 & .918 & & 21.032 \\
\hline \multirow[t]{3}{*}{ V } & 2012 & 96.000 & 126.000 & 103.160 & 26.980 & 10.330 & 10.330 & 239.530 & 44.330 & 1.840 & 61.560 & 26.400 & 15.070 & 43.680 & 33.430 & 6.000 & 2.070 & 2.940 & 4.110 & 21.710 \\
\hline & 2013 & 101.000 & 131.000 & 97.733 & 27.167 & 9.733 & 9.867 & 180.533 & 36.233 & 1.693 & 45.932 & 20.385 & 12.970 & 46.400 & 28.116 & 5.567 & 1.767 & 3.153 & 5.779 & 20.287 \\
\hline & pooled & 102.222 & 132.639 & 96.596 & 24.481 & 8.872 & 8.972 & 164.464 & 35.601 & 1.474 & 40.811 & 21.407 & 11.161 & 45.689 & 28.425 & 5.556 & 2.033 & 2.766 & 5.852 & 20.537 \\
\hline \multirow[t]{3}{*}{ VI } & 2012 & 105.670 & 135.670 & 104.470 & 26.320 & 9.300 & 9.800 & 175.530 & 26.500 & 1.670 & 33.190 & 25.550 & 16.800 & 53.670 & 31.400 & 6.570 & 2.200 & 2.990 & 5.280 & 21.990 \\
\hline & 2013 & 106.000 & 136.000 & 104.700 & 27.167 & 9.133 & 9.400 & 193.800 & 29.700 & 1.467 & 33.514 & 24.273 & 17.167 & 53.833 & 31.871 & 6.100 & 2.133 & 2.871 & 4.944 & 21.931 \\
\hline & pooled & 106.833 & 136.833 & 104.867 & 27.973 & 9.350 & 9.533 & 185.967 & 29.167 & 1.467 & 32.631 & 24.328 & 16.647 & 53.817 & 31.148 & 6.150 & 2.100 & 2.934 & 4.639 & 22.027 \\
\hline
\end{tabular}


Table.4 Contribution of different characters towards genetic divergence in rice

\begin{tabular}{|r|l|c|c|c|}
\hline S. No. & \multicolumn{1}{|c|}{ Character } & \multicolumn{3}{|c|}{ Contribution (per cent) } \\
\cline { 3 - 5 } & & $\mathbf{2 0 1 2}$ & $\mathbf{2 0 1 3}$ & Pooled \\
\hline $\mathbf{1}$ & Days to 50\% Flowering & 3.36 & 3.42 & 3.57 \\
\hline $\mathbf{2}$ & Days to Maturity & 2.64 & 2.66 & 2.79 \\
\hline $\mathbf{3}$ & Plant Height & 1.98 & 2.04 & 2.00 \\
\hline $\mathbf{4}$ & Panicle Length & 4.11 & 3.52 & 3.77 \\
\hline $\mathbf{5}$ & No. of Productive Tillers & 3.84 & 4.16 & 3.96 \\
\hline $\mathbf{6}$ & No. of Tiller & 3.79 & 4.16 & 3.89 \\
\hline $\mathbf{7}$ & No. of Grain Per Panicle & 10.01 & 11.35 & 11.20 \\
\hline $\mathbf{8}$ & Leaf Length & 6.90 & 5.86 & 7.05 \\
\hline $\mathbf{9}$ & Leaf Breadth & 4.78 & 4.53 & 4.44 \\
\hline $\mathbf{1 0}$ & Flag Leaf Area & 9.51 & 8.27 & 8.99 \\
\hline $\mathbf{1 1}$ & 1000 Grain Weight & 6.87 & 6.36 & 6.93 \\
\hline $\mathbf{1 2}$ & Grain Yield Per Plant & 6.36 & 7.37 & 7.08 \\
\hline $\mathbf{1 3}$ & Dry Weight & 6.59 & 5.66 & 5.28 \\
\hline $\mathbf{1 4}$ & Harvest Index & 5.72 & 7.45 & 4.71 \\
\hline $\mathbf{1 5}$ & Grain Length & 3.99 & 3.88 & 4.08 \\
\hline $\mathbf{1 6}$ & Grain Breadth & 3.86 & 3.27 & 3.59 \\
\hline $\mathbf{1 7}$ & L/B Ratio & 4.73 & 4.82 & 4.82 \\
\hline $\mathbf{1 8}$ & Alkali Digestion & 9.77 & 9.86 & 10.32 \\
\hline $\mathbf{1 9}$ & Amylose Content & 1.53 & 1.80 & 1.71 \\
\hline
\end{tabular}

The minimum inter-cluster distance was found between clusters II and III (11.411) during 2012, between clusters II and V (11.391) during 2013 and between clusters I and IV (7.984) in pooled estimates. This suggested that the lines belonging to these clusters were relatively closer to each other hence to maintain broad genetic base hybridization between lines of these clusters should be avoided.

The largest intra-cluster $\mathrm{D}^{2}$ values were recorded in cluster III (39.238), cluster I (45.769), cluster II (50.256) during 2012, 2013 and over the season respectively. The lines included in clusters III, I and II were relatively more diverse than those in the other clusters (Table 2). The diversity was also supported by the appreciable amount of variation among the cluster means for different characters.
During 2012 cluster I showed the maximum cluster means for number of grains per panicle and $\mathrm{L} / \mathrm{B}$ ratio, the lines from Cluster III had maximum means for dry weight and alkali digestion value. Cluster IV showed maximum means for $50 \%$ flowering and days to maturity. Cluster V showed the maximum cluster means for panicle length, number of productive tillers, flag leaf length, flag leaf breadth, flag leaf area, 1000 grain weight and harvest index. Cluster VI had maximum cluster means for plant height, grain yield per plant, grain length, grain breadth and amylose content. During 2013 cluster I had maximum means for $50 \%$ flowering, days to maturity, panicle length, dry weight per plant and alkali digestion value. Cluster III had maximum means for number of productive tillers per plant, number of tillers, number of grain per panicle, flag leaf length, flag leaf breadth, flag leaf area, 1000 grain weight and L/B ratio. 
Cluster IV had maximum means for plant height, grain yield per panicle, harvest index, grain length and amylose content. Cluster VI showed maximum means for grain breadth.

However, when data from both the seasons were pooled cluster I had maximum cluster mean value for flag leaf breadth. Cluster II had maximum mean values for number of productive tillers per plant, number of tillers per plant, flag leaf length, flag leaf area and L/B ratio. Cluster III showed maximum means for days to $50 \%$ flowering, days to maturity and harvest index. In cluster IV exhibited highest cluster mean values for number of grains per panicle. Cluster V had highest cluster mean value for alkali digestion value. In Cluster VI highest cluster mean values were observed for plant height, panicle length, 1000 grain weight, grain yield per plant, dry weight per plant, grain length, grain breadth and amylose content (Table 3 ).

The number of grains per panicle (10.01, 11.35, and 11.20) had maximum contribution and amylose content (1.53, 1.80, and 1.71) had minimum contribution towards diversity in both the season as well as over the seasons. 1000 grain weight, dry weight per plant, flag leaf area, flag leaf length and grain yield per plant showed moderate contribution towards diversity in both the seasons as well as over the seasons (Table 4).

In conclusion the considering the importance of genetic distance, relative contributions of characters towards total divergence and yield potential of genotypes, the present investigation suggests that, parental lines elected from cluster I (C-1446-5-18-17-1MLD 2, JGL-17183, WGL- 365, UPR-34111-1-1, UPR-3305-6-1-2, CR-77-3-1-1, NP107-5, GontraBidhan-3, UPR-3027-10-1-1) for number of productive tillers, cluster IV (UPRI- 3425-14-3-1, UPR-3443-7-2-1, NP218, KAR-559-1) number of grains per panicle and cluster VI (Pant Dhan-4) for panicle length and grain yield per plant could be used in crossing programmes to achieve desired segreants. Since No. of grains per panicle contributed maximum towards the genetic divergence, we may go for direct selection of this trait for diversity purpose.

On the basis of above observations, it is quite obvious that material have sufficient amount of diversity for desired traits. The diversity between clusters can be utilized for restoration of hybridization and trait selection related to divergence. Thus, it would be beneficial for selection of superior lines which could be used in hybridization programme.

\section{References}

Chaturvedi, H.P. and Maurya, D.M. (2005). Genetics divergence analysis in rice (Oryza sativa L.), Adv. Pl. Sci., 18(1): 349-353. Mahalanobis, P.C. (1936). On generalized distance in statistics. Proceedings of National Institute of Science, India, 2: 49-55.

Mishra, L.K., Sarawgi, A.K. and Mishra, R.K. (2003). Genetic diversity for morphological and quality traits in rice (Oryza sativa L.), Adv. in Pl. Sci., 16(1): 287-293.

Murty, B.R. and Arunachalam, V. (1967). Computer programmes for some problems in biometrical genetics-I. Use of Mahalanobis $\mathrm{D}^{2}$ in classification problem. Ind. J. Genet. Pl. Breed., 27:60-69.

Ramya, K. and Senthilkumar, K. (2008). Genetic divergence in rice. Crop Improv.35(2): 119-21.

Rao, C.R. (1952). The concept of distance and the problem of group constellation. In Advance Statistical Methods in Biometric Research. John Willey and Sons. Inc., New York, USA, pp. 351- 
378.

Rao, T.P., Gomathinayagam, P. (1997). Genetic diversity in semi dry rice under different environments. Madras Agr. J. 84, 314-317.

Sandhyakishore, N., Babu, V.R., Ansari, N.A. and Chandran, R. (2007). Genetics divergence analysis using yield and quality traits in rice (Oryza sativa L.), Crop Improv.,34:12-13.
Sarawgi, A.K. and Rita Binse. (2007). Studies on genetic divergence of aromatic rice germplasm for agro-morphological and quality characters. Oryza, 44(1): 74-76.

Singh, R. B., and Bains, S. S. (1968). Genetic divergence for ginning outturn and its components in upland cotton. Indian Journal of Genetics and Plant Breeding (The), 28(3), 262-268.

\section{How to cite this article:}

Aditya Bora, Dhanraj Meena, Mukesh Kumar Karnwal and Himanshu Chaudhary. 2020. Genetic Divergence for Important Economic and Quality Traits in Improved Lines of Rice (Oryza sativa L.). Int.J.Curr.Microbiol.App.Sci. 9(09): 3369-3376.

doi: https://doi.org/10.20546/ijcmas.2020.909.419 